\title{
An Arithmetic Characterization of Decomposition Methods in Banach Spaces Similar to Pełczyński's Decomposition Method
}

\author{
by \\ Elói Medina GALEGO \\ Presented by Stanistaw KWAPIEŃ
}

Summary. Inspired by Pełczyński's decomposition method in Banach spaces, we introduce the notion of Schroeder-Bernstein quadruples for Banach spaces. Then we use some Banach spaces constructed by W. T. Gowers and B. Maurey in 1997 to characterize them.

1. Introduction. For the sake of clarity we start with the notation. Let $X$ and $Y$ be Banach spaces. We write $X \stackrel{c}{\hookrightarrow} Y$ if $X$ is isomorphic to a complemented subspace of $Y$, and $X \sim Y$ if $X$ is isomorphic to $Y$. If $n \in \mathbb{N}^{*}$ $=\{1,2, \ldots\}$, then $X^{n}$ denotes the sum of $n$ copies of $X$. It is useful to define $X^{0}=\{0\}$. Finally, if $m, n$ are integer numbers, then $m \mid n$ means that $m$ divides $n$.

Suppose that $X$ and $Y$ are Banach spaces satisfying

$$
X \stackrel{c}{\hookrightarrow} Y, \quad Y \stackrel{c}{\hookrightarrow} X .
$$

In 1996 W. T. Gowers [6] solved the so-called Schroeder-Bernstein Problem for Banach spaces by showing that $X$ is not necessarily isomorphic to $Y$. However Pełczyński's decomposition method [1, p. 64] states that $X \sim Y$ if we add to (1) the two conditions

$$
X \sim X^{2}, \quad Y \sim Y^{2} .
$$

This decomposition method has played an important role in the isomorphic theory of classical Banach spaces (see [1]). So after the variety of solutions to the Schroeder-Bernstein Problem (see [2]-[7]) it is natural to ask whether

2000 Mathematics Subject Classification: Primary 46B03, 46B20.

Key words and phrases: Pełczyński's decomposition method, Schroeder-Bernstein problem. 
it is possible to determine all pairs of non-trivial conditions similar to those of (2) which added to (1) also yield $X \sim Y$. To be more precise we define:

Definition 1.1. We say that a quadruple $(p, q, r, s)$ of natural numbers with $p+q \geq 2$ and $r+s \geq 2$ is a Schroeder-Bernstein quadruple (SBQ) if $X \sim Y$ for any Banach spaces $X$ and $Y$ satisfying (1) and the conditions

$$
X \sim X^{p} \oplus Y^{q}, \quad Y \sim X^{r} \oplus Y^{s} .
$$

The restrictions $p+q \geq 2$ and $r+s \geq 2$ are imposed to avoid the conditions $X \sim\{0\}, Y \sim\{0\}, X \sim X, Y \sim Y$ and $X \sim Y$ in (3).

We also say that $\Delta=(p-1)(s-1)-r q$ is the discriminant of the quadruple $(p, q, r, s)$.

Thus Pełczyński's decomposition method states that $(2,0,0,2)$ is a SBQ. The aim of this paper is to present a simple characterization of the SBQ in terms of their discriminant. To do this, the Banach spaces constructed by W. T. Gowers and B. Maurey in [7, p. 563] will be fundamental (see Remark 2.4).

2. The result. Our characterization of the SBQ is given by Theorem 2.1. It follows directly from Propositions 2.5 and 2.6.

TheOREM 2.1. A quadruple $(p, q, r, s)$ of natural numbers with $p+q \geq 2$ and $r+s \geq 2$ is a $S B Q$ if and only if $\Delta \neq 0, \Delta \mid(p+q-1)$ and $\Delta \mid(r+s-1)$.

In order to prove our propositions, we need some auxiliary results. We begin with a simple remark which will be used several times in this work.

Remark 2.2. Let $X$ and $Y$ be Banach spaces and $(p, q, r, s)$ a quadruple of natural numbers with $p+q \geq 2$ and $r+s \geq 2$, satisfying (3). Then the discriminant of $(s, r, q, p)$ is also $\Delta$ and

$$
Y \sim Y^{s} \oplus X^{r}, \quad X \sim Y^{q} \oplus X^{p} .
$$

Lemma 2.3. Let $(p, q, r, s)$ be a quadruple of natural numbers with $p>r$, $q>s, r+s \geq 2, \Delta \mid(p+q-1)$ and $\Delta \mid(r+s-1)$. Suppose that $X$ and $Y$ are Banach spaces satisfying (3). Then there exist $P, Q, R, S \in \mathbb{N}$ such that either $P \geq R$ and $Q \leq S$, or $P \leq R$ and $Q \geq S$, satisfying

(a) $P+Q \geq 2$ and $R+S \geq 2$.

(b) The discriminant of $(P, Q, R, S)$ is $\Delta$.

(c) $\Delta \mid(P+Q-1)$ and $\Delta \mid(R+S-1)$.

(d) $X \sim X^{P} \oplus Y^{Q}$ and $Y \sim X^{R} \oplus Y^{S}$.

Proof. Let us start with two claims.

Claim 1. Put $R=r$ and $S=s$. Then there exist $P, Q \in \mathbb{N}$, with $P \leq R$ or $Q \leq S$, satisfying (a)-(d) of Lemma 2.3.

Indeed, we distinguish three cases: $s=0, s=1$ and $s>1$. 
CASE 1: $s=0$. Then $\Delta=-p+1-r q$ and $r \geq 2$. We write $p=r m+n$ for some $m \in \mathbb{N}^{*}$ and $0 \leq n<r$. By $(3), Y \sim X^{r}$ and

$$
X \sim\left(X^{r}\right)^{m} \oplus X^{n} \oplus Y^{q} \sim Y^{m} \oplus X^{n} \oplus Y^{q} \sim X^{n} \oplus Y^{q+m} .
$$

Put $P=n$ and $Q=q+m$. Thus $P<R$ and according to (3) and (4), (d) is satisfied. Moreover,

(a) $P+Q=n+q+m \geq n+1+1 \geq 2$, because $q>s=0$ and $m \in \mathbb{N}^{*}$.

(b) The discriminant of $(P, Q, R, S)$ is equal to $-(n-1)-r(q+m)=$ $-(p-r m-1)-r(q+m)=-p+1-r q=\Delta$.

(c) Since $P+Q-1=n+(q+m)-1=(p-r m)+(q+m)-1=$ $(p+q-1)-m(r-1)$ and by hypothesis $\Delta \mid(p+q-1)$ and $\Delta \mid(r-1)$, it follows that $\Delta \mid(P+Q-1)$.

So in this case Claim 1 is proved.

CASE 2: $s=1$. Hence $\Delta=-r q$ and $q>s=1$. We write $p=r m+n$ for some $m \in \mathbb{N}^{*}$ and $0 \leq n<r$. By $(3), Y \sim X^{r} \oplus Y$ and

$$
\begin{aligned}
X \sim X^{r m+n} \oplus Y^{q} & \sim X^{r} \oplus X^{r} \oplus \cdots \oplus X^{r} \oplus Y \oplus X^{n} \oplus Y^{q-1} \\
& \sim X^{n} \oplus Y^{q} .
\end{aligned}
$$

Put $P=n$ and $Q=q$. Thus $P<R$ and according to (3) and (5), (d) is satisfied. Moreover,

(a) $P+Q=n+q \geq n+2 \geq 2$.

(b) The discriminant of $(P, Q, R, S)$ is equal to $-r q=\Delta$.

(c) Since $P+Q-1=n+q-1=(p-r m)+q-1=(p+q-1)-r m$ and by hypothesis $\Delta \mid(p+q-1)$ and $\Delta \mid r$, it follows that $\Delta \mid(P+Q-1)$.

Therefore in this case Claim 1 is also proved.

CASE 3: $s>1$.

STEP 3.1. Put $p_{1}=p-r>0$ and $q_{1}=q-s>0$. By (3) we have

$$
X \sim X^{p_{1}+r} \oplus Y^{q_{1}+s} \sim X^{r} \oplus Y^{s} \oplus X^{p_{1}} \oplus Y^{q_{1}} \sim X^{p_{1}} \oplus Y^{q_{1}+1} .
$$

Subcase 3.1.1: $p_{1} \leq r$. Put $P=p_{1}$ and $Q=q_{1}+1$. Thus $P \leq R$ and according to (3) and (6), (d) is satisfied. Moreover,

(a) $P+Q=p_{1}+q_{1}+1 \geq 1+1+1=3$.

(b) The discriminant of $(P, Q, R, S)$ is equal to $\left(p_{1}-1\right)(s-1)-r\left(q_{1}+1\right)=$ $(p-r-1)(s-1)-r(q-s+1)=p s-p-s+1-r q=\Delta$.

(c) Since $P+Q-1=p_{1}+\left(q_{1}+1\right)-1=(p-r)+(q-s)=$ $(p+q-1)-(r+s-1)$ and by hypothesis $\Delta \mid(p+q-1)$ and $\Delta \mid(r+s-1)$, it follows that $\Delta \mid(P+Q-1)$.

Hence in this subcase Claim 1 is proved. 
SubCASE 3.1.2: $q_{1}+1 \leq s$. Put $P=p_{1}$ and $Q=q_{1}+1$. Thus $Q \leq S$ and according to (3) and (6), (d) is satisfied. Now, from what we have just done in Subcase 3.1.1, Claim 1 is also proved.

Otherwise

$$
p_{1}>r, \quad s<q_{1}+1=q-(s-1) .
$$

SteP 3.2. Put $p_{2}=p_{1}-r>0$ and $q_{2}=q_{1}+1-s>0$. By (6) we have

$$
X \sim X^{p_{2}+r} \oplus Y^{q_{2}+s} \sim X^{r} \oplus Y^{s} \oplus X^{p_{2}} \oplus Y^{q_{2}} \sim X^{p_{2}} \oplus Y^{q_{2}+1} .
$$

Subcase 3.2.1: $p_{2} \leq r$. Put $P=p_{2}$ and $Q=q_{2}+1$. Thus $P \leq R$ and according to (3) and (7), (d) is satisfied. The remaining assertions of Claim 1 are proved similarly to Subcase 3.1.1.

Subcase 3.2.2: $q_{2}+1 \leq s$. Put $P=p_{2}$ and $Q=q_{2}+1$. Thus $Q \leq S$ and according (3) and (7), (d) is satisfied. The remaining assertions of Claim 1 are proved as in Subcase 3.1.2.

Otherwise

$$
p_{2}>r, \quad s<q_{2}+1=q-2(s-1) .
$$

Since $s>1$, after a finite number of steps Claim 1 is proved.

Next, note that thanks to Remark 2.2, it follows from Claim 1 that the following claim is also true:

Claim 2. Let $\left(p^{\prime}, q^{\prime}, r^{\prime}, s^{\prime}\right)$ be a quadruple of natural numbers with discriminant $\Delta, p^{\prime}<r^{\prime}, q^{\prime}<s^{\prime}, p^{\prime}+q^{\prime} \geq 2, \Delta \mid\left(p^{\prime}+q^{\prime}-1\right)$ and $\Delta \mid\left(r^{\prime}+s^{\prime}-1\right)$. Put $P=p^{\prime}$ and $Q=q^{\prime}$ and suppose that $X$ and $Y$ are Banach spaces satisfying

$$
X \sim X^{p^{\prime}} \oplus Y^{q^{\prime}}, \quad Y \sim X^{r^{\prime}} \oplus Y^{s^{\prime}} .
$$

Then there exist $R, S \in \mathbb{N}$ such that $P \geq R$ or $Q \geq S$ and satisfying (a)-(d) of Lemma 2.3 .

Now we are ready to prove Lemma 2.3.

SteP 1. Put $R_{1}=r$ and $S_{1}=s$. By Claim 1, there exist $P_{1}, Q_{1} \in \mathbb{N}$, with $P_{1} \leq R_{1}$ or $Q_{1} \leq S_{1}$, such that the quadruple $\left(P_{1}, Q_{1}, R_{1}, S_{1}\right)$ satisfies (a)-(c) of Lemma 2.3 and

$$
X \sim X^{P_{1}} \oplus Y^{Q_{1}}, \quad Y \sim X^{R_{1}} \oplus Y^{S_{1}} .
$$

If $P_{1}=R_{1}$ or $Q_{1}=S_{1}$ then Lemma 2.3 is proved.

Otherwise $P_{1}<R_{1}$ and $Q_{1}<S_{1}$.

SteP 2. Put $P_{2}=P_{1}$ and $Q_{2}=Q_{1}$. By Claim 2 applied in (8), there exist $R_{2}, S_{2} \in \mathbb{N}$, with $P_{2} \geq R_{2}$ or $Q_{2} \geq S_{2}$, such that the quadruple $\left(P_{2}, Q_{2}, R_{2}, S_{2}\right)$ satisfies (a)-(c) of Lemma 2.3 and

$$
X \sim X^{P_{2}} \oplus Y^{Q_{2}}, \quad Y \sim X^{R_{2}} \oplus Y^{S_{2}} .
$$

If $P_{2}=R_{2}$ or $Q_{2}=S_{2}$ then Lemma 2.3 is also proved. 
Otherwise $P_{2}>R_{2}$ and $Q_{2}>S_{2}$.

Finally observe that $R_{2}<P_{2}=P_{1}<R_{1}=r$. So after a finite number of steps Lemma 2.3 is proved.

REMARK 2.4. Let $p, q, r, s \in \mathbb{N}$ with $p+q \geq 2$ and $r+s \geq 2$. Suppose that there exists a quintuple $(i, j, k, l, t)$, with $i, j \in \mathbb{N}^{*}, k, l, t \in \mathbb{N}$ and $t \geq 2$, such that $t$ does not divide $j-i$,

$$
(p-1) i+q j=k t, \quad r i+(s-1) j=l t .
$$

Then $(p, q, r, s)$ is not a SBQ.

Indeed, let $X_{t}$ be the Banach space constructed by W. T. Gowers and B. Maurey in [7, p. 563], that is, $X_{t}^{u} \sim X_{t}^{v}$, with $u, v \in \mathbb{N}^{*}$, if and only if $u$ is equal to $v$ modulo $t$. By (9) we have

$$
X_{t}^{i} \sim\left(X_{t}^{i}\right)^{p} \oplus\left(X_{t}^{j}\right)^{q}, \quad X_{t}^{j} \sim\left(X_{t}^{i}\right)^{r} \oplus\left(X_{t}^{j}\right)^{s} .
$$

Furthermore, since $t$ does not divide $j-i$, it follows that $X_{t}^{i} \nsim X_{t}^{j}$. Therefore $(p, q, r, s)$ is not a SBQ.

Proposition 2.5. If a quadruple $(p, q, r, s)$ of natural numbers with $p+$ $q \geq 2$ and $r+s \geq 2$ is a $S B Q$, then $\Delta \neq 0, \Delta \mid(p+q-1)$ and $\Delta \mid(r+s-1)$.

Proof. First note that it is enough to show:

(a) If $\Delta=0$ then $(p, q, r, s)$ is not a SBQ.

(b) If $\Delta \neq 0$ and $\Delta$ does not divide $p+q-1$, then $(p, q, r, s)$ is not a SBQ.

(c) If $\Delta \neq 0$ and $\Delta$ does not divide $r+s-1$, then $(p, q, r, s)$ is not a SBQ.

We will prove (a) and (b). By symmetry, (c) is also true.

(a) Suppose $\Delta=0$. Since $p+q \geq 2$, there are three cases: $p=1, q=1$, $p \geq 2$ and finally $q \geq 2$.

CAsE 1: $p=q=1$. By the definition of $\Delta, r=0$ and therefore $s \geq 2$.

SubcASE 1.1: $s=2$. Let $X_{t}$ be the Banach space mentioned in Remark 2.4, with $t=2$. We take $X=X_{t}$ and $Y=X_{t}^{2}$. Hence (1) and (3) are satisfied, but $X \not Y$. Thus $(p, q, r, s)$ is not a SBQ.

SubCASE 1.2: $s \geq 3$. Let $X_{t}$ be as in Remark 2.4, with $t=s-1$. We take $X=X_{t}$ and $Y=X_{t}^{s-1}$. Therefore (1) and (3) are satisfied, but $X \nsim Y$. This implies that $(p, q, r, s)$ is not a SBQ .

CAse $2: p \geq 2$. Since $\Delta=0,(p, p-1, p-1, r, p+q)$ satisfies (9). So $(p, q, r, s)$ is not a SBQ, because $p+q$ does not divide 1 .

CASE 3: $q \geq 2$. Since $\Delta=0,(q, q+1, q, s-1, p+q)$ satisfies (9). Since $p+q$ does not divide $1,(p, q, r, s)$ is not a SBQ.

(b) Assume that $\Delta \neq 0$ and $\Delta$ does not divide $p+q-1$. Then $\Delta \neq 1$ and we consider the following two cases: $\Delta \geq 2$ and $\Delta \leq-2$. 
CASE 1: $\Delta \geq 2$. It follows from the definition of $\Delta$ that $s>1$. There are two subcases: $r>0$ and $r=0$.

SubcASE 1.1: $r>0$. Since $\Delta>0, q /(s-1)<(p-1) / r$. Let $m, n \in \mathbb{N}^{*}$ be such that

$$
q /(s-1)<m / n<(p-1) / r .
$$

Here we also distinguish two possibilities:

SubCASE 1.1.1: $\Delta \nmid(m(r+s-1)-n(p+q-1))$. Let $i=m(s-1)-n q$ and $j=n(p-1)-m r$. From the inequalities in (10), we have $i>0$ and $j>0$. It is easy to verify that $(i, j, m, n, \Delta)$ satisfies (9). Furthermore, $\Delta$ does not divide $j-i=n(p+q-1)-m(r+s-1)$. Consequently, $(p, q, r, s)$ is not a $\mathrm{SBQ}$.

Subcase 1.1.2: $\Delta \mid(m(r+s-1)-n(p+q-1))$. Since $(v m) /(v n+1)$ tends to $m / n$ as $v \rightarrow \infty$, there exists $v \in \mathbb{N}^{*}$ such that

$$
q /(s-1)<(v m) /(v n+1)<(p-1) / r .
$$

We take $i=v m(s-1)-(v n+1) q$ and $j=(v n+1)(p-1)-v m r$. By (11) we have $i>0$ and $j>0$. Now observe that $(i, j, v m, v n, \Delta)$ satisfies (9). But, of course, $\Delta$ does not divide $j-i=v n(p+q-1)-v m(r+s-1)+p+q-1$, hence $(p, q, r, s)$ is not a SBQ.

SubCASE 1.2: $r=0$. By the definition of $\Delta, s \geq 2$. To show that $(p, q, r, s)$ is not a SBQ, it suffices to take $m, n \in \mathbb{N}^{*}$ such that $q /(s-1)<$ $m / n$ and to proceed as in Subcase 1.1. Only observe that here we must consider separately the cases when $\Delta$ does or does not divide $m(s-1)-$ $n(p+q-1)$.

CAsE 2: $\Delta \leq-2$. In this case, it is useful to distinguish the following four possible subcases: $s=0, r=0, s>1$ and $r>0$, and finally $s=1$.

Subcase 2.1: $s=0$. Thus $r \geq 2$ and $-\Delta=(p-1)+r q \geq 2$. Since $-\Delta$ does not divide $p+q-1, q \geq 1$. Therefore $(1, r, 1,0,-\Delta)$ satisfies $(9)$. Moreover, $-\Delta=(p+q-1)+(r-1) q$, hence $-\Delta$ does not also divide $r-1$. So $(p, q, r, s)$ is not a SBQ.

SubcASE 2.2: $r=0$. Therefore $\Delta=(p-1)(s-1) \leq-2$. Since $s \geq 2$, we have $p=0$. Hence $(q, 1,0,1,-\Delta)$ satisfies (9). Thus, $(p, q, r, s)$ is not a SBQ, because by hypothesis $\Delta$ does not divide $q-1$.

SubCASE 2.3: $s>1$ and $r>0$. Since $\Delta<0,(p-1) / r<q /(s-1)$. Now replacing $\Delta$ by $-\Delta$, by an analogous argument to that used in Case 1 , we deduce that $(p, q, r, s)$ is not a SBQ.

SubCASE 2.4: $s=1$. So $r \geq 1, \Delta=-r q \leq-2$ and therefore $q \geq 1$. It suffices to take $m, n \in \mathbb{N}^{*}$ such that $(p-1) / r<m / n$ and replacing $\Delta$ by $-\Delta$ to proceed as in Subcase 1.2 to see that $(p, q, r, s)$ is not a SBQ. 
Proposition 2.6. Suppose that $(p, q, r, s)$ is a quadruple of natural numbers with $p+q \geq 2, r+s \geq 2, \Delta \neq 0, \Delta \mid(p+q-1)$ and $\Delta \mid(r+s-1)$. Then $(p, q, r, s)$ is a $S B Q$.

Proof. Let $X$ and $Y$ be Banach spaces satisfying (1) and (3). We will prove that $X \sim Y$ by considering the following six possible cases: $p=r$ and $s>q ; p>r$ and $s=q ; p>r$ and $s>q ; p \leq r$ and $s \leq q ; p>r$ and $s<q$; and finally $p<r$ and $s>q$.

CASE 1: $p=r$ and $s>q$. In this case it is also convenient to consider three subcases: $r=0, r=1$ and $r>1$.

SubCASE 1.1: $r=0$. Thus $\Delta=-(s-1)$ and by hypothesis there exists $v \in \mathbb{N}^{*}$ such that $q-1=v(s-1)$. By $(3), Y \sim Y^{s}$ and

$$
X \sim Y^{q-1} \oplus Y \sim Y^{v(s-1)} \oplus Y \sim Y^{s-1} \oplus Y^{s-1} \oplus \cdots \oplus Y^{s-1} \oplus Y \sim Y .
$$

Subcase 1.2: $r=1$. Hence $\Delta=-q$ and thus there exists $v \in \mathbb{N}^{*}$ such that $s=v q$. By $(3), X \sim X \oplus Y^{q}$ and

$$
Y \sim X \oplus Y^{s} \sim X \oplus Y^{v q} \sim X \oplus Y^{q} \oplus Y^{q} \oplus \cdots \oplus Y^{q} \sim X .
$$

SubCASE 1.3: $r>1$. Let $D \in \mathbb{N}^{*}$ be such that $s=q+D$. Thus

$$
\Delta=(r-1)(D-1)-q \text {. }
$$

Then, using (3), we have

$$
Y \sim X^{r} \oplus Y^{q+D} \sim\left(X^{r} \oplus Y^{q}\right) \oplus Y^{D} \sim X \oplus Y^{D}
$$

Adding $X \oplus Y^{D-1}$ to both sides of (13) we deduce that

$$
Y \sim X^{2} \oplus Y^{D+(D-1)} .
$$

Hence, by induction we obtain

$$
Y \sim X^{r} \oplus Y^{D+(r-1)(D-1)}
$$

To continue we distinguish two possibilities: $\Delta<0$ and $\Delta>0$.

SubCASE 1.3.1: $\Delta<0$. By our hypothesis, $-\Delta \mid D$. Therefore there exists $v \in \mathbb{N}^{*}$ satisfying $D=-v \Delta$. Consequently, by (3), (12) and (14) we conclude that

$$
Y \sim X^{r} \oplus Y^{(r-1)(D-1)-\Delta} \oplus Y^{D+\Delta} \sim X^{r} \oplus Y^{q} \oplus Y^{D+\Delta} \sim X \oplus Y^{D+\Delta} .
$$

Again by induction, we get $Y \sim X \oplus Y^{D+m \Delta}$ for every $m \in \mathbb{N}^{*}, m \leq v$. In particular, $Y \sim X \oplus Y^{D+v \Delta} \sim X$.

Subcase 1.3.2: $\Delta>0$. Let $v \in \mathbb{N}^{*}$ be such that $D=v \Delta$. According to $(3),(12)$ and $(14)$ we have

$$
Y \sim X^{r} \oplus Y^{(r-1)(D-1)-\Delta} \oplus Y^{D+\Delta} \sim X^{r} \oplus Y^{q} \oplus Y^{D+\Delta} \sim X \oplus Y^{D+\Delta} .
$$

Therefore, once more, by induction it is easy to see that

$$
Y \sim X \oplus Y^{D+v \Delta} \sim X \oplus Y^{2 D}
$$


Thus, it follows from (13) and (15) that

$$
Y \sim X \oplus Y^{D} \oplus Y^{D} \sim Y^{D+1} .
$$

Let $A$ be a Banach space satisfying

$$
X \sim Y \oplus A .
$$

So, again by (13) and (16),

$$
X \sim Y \oplus A \sim Y^{D+1} \oplus A \sim Y \oplus A \oplus Y^{D} \sim X \oplus Y^{D} \sim Y .
$$

CASE 2: $p>r$ and $s=q$. By Remark 2.2 and Case 1, it follows that $X \sim Y$.

CASE 3: $p>r$ and $s>q$. Then $\Delta>0$. Let $d$ and $D$ in $\mathbb{N}^{*}$ be such that $p=r+d$ and $s=q+D$. Thus

$$
\Delta=r(D-1)+q(d-1)+(d-1)(D-1) .
$$

There are two subcases: $\Delta=1$ and $\Delta \geq 2$.

SUBCASE 3.1: $\Delta=1$. Hence only one of the three summands in (18) is not zero and equal to 1 . There are three possibilities to consider: $r(D-1)=1$, $q(d-1)=1$ and $(d-1)(D-1)=1$.

SubCASE 3.1.1: $r(D-1)=1$. So $r=1$ and $D=2$. Consequently, by (18), $d=1, p=2$ and $q$ is arbitrary. Now by using (3) and (17) we have

$$
X \sim X \oplus Y^{q+1} \oplus Y \oplus A \sim X \oplus Y^{q+1} \oplus X \sim X^{2} \oplus Y^{q+1} .
$$

Let $B$ be a Banach space satisfying

$$
Y \sim X \oplus B
$$

Hence, again by (3),

(21) $Y \sim X^{2} \oplus Y^{q} \oplus B \sim X \oplus Y^{q} \oplus X \oplus B \sim X \oplus Y^{q} \oplus Y \sim X \oplus Y^{q+1}$.

Therefore from (19) and (21), $X \sim X \oplus Y$. Finally, again from (21), we get

$$
Y \sim X \oplus Y^{q+1} \sim X \oplus Y \oplus Y \oplus \cdots \oplus Y \sim X .
$$

Subcase 3.1.2: $q(d-1)=1$. So $q=1$ and $d=2$. Therefore by (18), $D=1$ and $r$ is arbitrary. By Remark 2.2 and Subcase 3.1.1, it follows that $X \sim Y$.

Subcase 3.1.3: $(d-1)(D-1)=1$. So $d=2$ and $D=2$. Thus by (18), $r=0$ and $q=0$. Consequently, Pełczyński's decomposition method implies that $X \sim Y$.

SubCase 3.2: $\Delta \geq 2$. So $p>1$. By (18) and our hypothesis we have

$$
\begin{aligned}
& (r(D-1)+q(d-1)+(d-1)(D-1)) \mid(r+d+q-1), \\
& (r(D-1)+q(d-1)+(d-1)(D-1)) \mid(r+q+D-1) .
\end{aligned}
$$

There are two possibilities: $D-1=0$ and $D-1>0$. 
Subcase 3.2.1: $D-1=0$. Then $(22)$ and (23) can be rewritten respectively as follows:

$$
q(d-1)|(r+d+q-1), \quad q(d-1)|(r+q) .
$$

Therefore $q(d-1) \mid(d-1)$. Consequently, $q=1$ and $d-1 \geq 2$, because $\Delta \geq 2$. Also by $(24)$ we conclude that $(d-1) \mid(r+1)$, that is, $(p-r-1) \mid(r+1)$.

Since $D=1$ and $s=q+D=2$, it follows from (3) and (17) that

$$
X \sim Y \oplus A \sim X^{r} \oplus Y^{2} \oplus A \sim X^{r} \oplus Y \oplus Y \oplus A \sim X^{r+1} \oplus Y .
$$

Now let $v \in \mathbb{N}^{*}$ be such that $r+1=v(p-r-1)$. Thus, (3) and (19) imply that

$$
Y \sim X \oplus B \sim X^{p} \oplus Y \oplus B \sim X^{p-1} \oplus Y \oplus X \oplus B \sim X^{p-1} \oplus Y^{2} .
$$

Hence, according to (25), we have $Y \sim X^{p-1} \oplus Y^{2} \sim X^{(p-1)-(r+1)} \oplus Y \oplus$ $Y \oplus X^{r+1} \sim X^{(p-1)-(r+1)} \oplus Y \oplus X \sim X^{p-r-1} \oplus Y$. Finally, again by $(25)$, we deduce

$$
X \sim X^{v(p-r-1)} \oplus Y \sim X^{p-r-1} \oplus X^{p-r-1} \oplus \ldots \oplus X^{p-r-1} \oplus Y \sim Y .
$$

SubCase 3.2.2: $D-1>0$. Then $d-1=0$, otherwise by (18), $\Delta>$ $(r+q+D-1)$ and thus (23) would not be true. Hence, by Remark 2.2 and Subcase 3.2.1, it follows that $X \sim Y$.

CASE $4: p \leq r$ and $s \leq q$. Here it is convenient to distinguish three subcases: $p \geq 1$ and $s \geq 1 ; p=0$; and finally $s=0$.

SubCase 4.1: $p \geq 1$ and $s \geq 1$. Then, from (3), (17) and (20), we obtain

$$
\begin{aligned}
& X \sim X^{r} \oplus Y^{s-1} \oplus Y \oplus A \sim X^{r} \oplus Y^{s-1} \oplus X \sim X^{r+1} \oplus Y^{s-1}, \\
& Y \sim X^{p-1} \oplus Y^{q} \oplus X \oplus B \sim X^{p-1} \oplus Y^{q} \oplus Y \sim X^{p-1} \oplus Y^{q+1} .
\end{aligned}
$$

In order to see that $X \sim Y$, it suffices to apply Case 3 in (26) and (27). Because $(r+1)+(s-1)-1=r+s-1,(p-1)+(q+1)-1=p+q-1$, the discriminant of $(r+1, s-1, p-1, q+1)$ is equal to $-\Delta, r+1>p-1$ and $q+1>s-1$.

Subcase 4.2: $p=0$. Thus $q \geq 2$ and by hypothesis we know that

$$
((s-1)+r q) \mid(q-1) \text {. }
$$

Therefore $r \leq 1$. If $r=1$, then again by (28), $s=0$, which is absurd, because $r+s \geq 2$. So, $r=0$ and (28) implies that there exists $v \in \mathbb{N}^{*}$ such that $q-1=v(s-1)$. Consequently, as in Subcase 1.1, we can show that $X \sim Y$.

SubCASE 4.3: $s=0$. By Remark 2.2 and Subcase 4.2, it follows that $X \sim Y$.

CASE 5: $p>r$ and $s<q$. By Lemma 2.3, there exist $P, Q, R, S \in \mathbb{N}$ satisfying (a)-(d) of that lemma and such that either $P \geq R$ and $Q \leq S$, or $P \leq R$ and $Q \geq S$. That is, exactly one of the following four possibilities is 
satisfied: $P=R$ and $S>Q ; P>R$ and $S=Q ; P>R$ and $S>Q ; P \leq R$ and $S \leq Q$. Therefore, from Cases 1-4, we see that $X \sim Y$.

CASE 6: $p<r$ and $s>q$. By Remark 2.2 and Case 5, it follows that $X \sim Y$.

This finishes the proof of Proposition 2.6.

Corollary 2.7. Suppose that $X$ and $Y$ are Banach spaces satisfying (1) and $X \sim X^{p}$ for some $p \in \mathbb{N}, p \geq 2$. Then $X \sim Y$ if and only if $Y \sim X^{r} \oplus Y^{2}$ for some $r \in \mathbb{N}$ with $(p-1) \mid(r+1)$.

Problem 2.8. Suppose that $X$ and $Y$ are Banach spaces satisfying (1) and $Y \sim X^{r} \oplus Y^{2}$ for some $r \in \mathbb{N}^{*}$. Does it follow that $X \sim X^{p}$ for some $p \in \mathbb{N}, p \geq 2$ ?

Finally, note that Theorem 2.1 gives a partial affirmative answer to the following problem:

Problem 2.9. Suppose that $X$ and $Y$ are Banach spaces satisfying (1) and (3) for some $p, q, r, s \in \mathbb{N}$ with $p+q \geq 2, r+s \geq 2$. Is it true that there exists $m \in \mathbb{N}, m \geq 2$, such that $X \sim X^{m}$ or $Y \sim Y^{m}$ ?

\section{References}

[1] P. G. Casazza, The Schroeder-Bernstein property of Banach space, in: Contemp. Math. 85, Amer. Math. Soc., 1989, 61-77.

[2] E. M. Galego, How to generate new Banach spaces non-isomorphic to their cartesian squares, Bull. Polish Acad. Sci. Math. 47 (1999), 21-25.

[3] - Banach spaces complemented in each other without isomorphic finite sums, ibid. 50 (2002), 1-9.

[4] -, On solutions to the Schroeder-Bernstein problem for Banach spaces, Arch. Math. (Basel) 74 (2002), 299-307.

[5] W. T. Gowers, A solution to the Schroeder-Bernstein problem for Banach space, preliminary version (unpublished).

[6] -, A solution to the Schroeder-Bernstein problem for Banach space, Bull. London Math. Soc. 28 (1996), 297-304.

[7] W. T. Gowers and B. Maurey, Banach spaces with small spaces of operators, Math. Ann. 307 (1997), 543-568.

Elói Medina Galego

Department of Mathematics

IME, University of São Paulo

São Paulo, 05315-970 Brazil

E-mail: eloi@ime.usp.br

Received September 25, 2003;

received in final form June 6, 2004 